\title{
Single Event Effect Characterization of the Mixed-Signal ASIC Developed for CCD Camera in Space Use
}

\author{
Hiroshi Nakajima ${ }^{\mathrm{a}, *}$, Mari Fujikawa ${ }^{\mathrm{a}}$, Hideki Mori ${ }^{\mathrm{a}}$, Hiroaki Kan ${ }^{\mathrm{a}}$, Shutaro Ueda ${ }^{\mathrm{a}}$, Hiroko Kosugi ${ }^{\mathrm{a}}$, Naohisa Anabuki ${ }^{\mathrm{a}}$, \\ Kiyoshi Hayashida ${ }^{\mathrm{a}}$, Hiroshi Tsunemi ${ }^{\mathrm{a}}$, John P. Doty ${ }^{\mathrm{b}}$, Hirokazu Ikeda ${ }^{\mathrm{c}}$, Hisashi Kitamura ${ }^{\mathrm{d}}$, and Yukio Uchihori ${ }^{\mathrm{d}}$ \\ ${ }^{a}$ Department of Earth and Space Science, Graduate School of Science, Osaka University, 1-1 Machikaneyama, Toyonaka, Osaka, \\ 560-0043, Japan \\ ${ }^{b}$ Noqsi Aerospace Ltd., @2822 South Nova Road, Pine, Colorado 80470, USA \\ ${ }^{c}$ the Institute of Space and Astronautical Science, Japan Aerospace Exploration Agency, 3-1-1 Yoshinodai, Chuo-ku, Sagamihara, \\ Kanagawa, 252-5210, Japan \\ ${ }^{d}$ National Institute for Radiological Sciences (NIRS), Anagawa 4-9-1, Inage-ku, Chiba-shi, Chiba, 263-8555, Japan
}

\begin{abstract}
We present the single event effect (SEE) tolerance of a mixed-signal application-specific integrated circuit (ASIC) developed for a charge-coupled device camera onboard a future X-ray astronomical mission. We adopted proton and heavy ion beams at HIMAC/NIRS in Japan. The particles with high linear energy transfer $(\mathrm{LET}) \mathrm{of} 57.9 \mathrm{MeV} \cdot \mathrm{cm}^{2} / \mathrm{mg}$ is used to measure the single event latch-up (SEL) tolerance, which results in a sufficiently low cross-section of $\sigma_{\mathrm{SEL}}$ $<4.2 \times 10^{-11} \mathrm{~cm}^{2} /($ Ion $\times$ ASIC). The single event upset (SEU) tolerance is estimated with various kinds of species with wide range of energy. Taking into account that a part of the protons creates recoiled heavy ions that has higher LET than that of the incident protons, we derived the probability of SEU event as a function of LET. Then the SEE event rate in a low-earth orbit is estimated considering a simulation result of LET spectrum. SEL rate is below once per 49 years, which satisfies the required latch-up tolerance. The upper limit of the SEU rate is derived to be $1.3 \times 10^{-3}$ events/sec. Although the SEU events cannot be distinguished from the signals of X-ray photons from astronomical objects, the 'derived SEU rate is below $1.3 \%$ of expected non X-ray background rate of the detector and hence these events should not be a major component of the instrumental background.
\end{abstract}

Keywords: X-ray, ASIC, CCD camera, Single Event Effect $2000 \mathrm{MSC}$ :

\section{Introduction}

The X-ray charge-coupled device (CCD) cameras have ${ }_{21}^{20}$ been functioning as the primary focal plane detector of ${ }_{22}$ modern X-ray astronomy thanks to their well-balanced ${ }_{23}^{22}$ performances for imaging-spectroscopy [1, 2, 3]. Although ${ }_{24}$ we owe the imaging performance of a camera to the $\mathrm{X}-{ }_{25}$ ray telescope, the energy and timing resolutions depend ${ }_{26}$ heavily both on the readout noise and on the processing ${ }_{27}^{26}$ speed of the front-end electronics, respectively. Since the ${ }_{28}$ forthcoming camera systems will require a larger number ${ }_{20}$ of pixels, a higher frame rate and a lower readout noise ${ }_{30}$ than those of the conventional systems, implementing the electronics with discrete integrated circuits (ICs) will dis- ${ }_{32}$ sipate too much power in orbit.

Recently, application-specific integrated circuits (ASICs) have been applied to the readout systems of the detectors including X-ray CCDs [4, 5, 6]. Drastically curtailed ${ }_{36}$ power consumption and compact size allow us to equip ${ }_{37}$

*Further author information: (Send correspondence to H.N.) H.N.: E-mail: nakajima@ess.sci.osaka-u.ac.jp, Telephone: $+816^{40}$ 68505478 many chips next to the sensors. Hence we have developed the mixed-signal Complementary Metal Oxide Semiconductor (CMOS) ASIC as the signal processing electronics for CCD cameras in space use [7, 8]. It is characterized with not only a low-noise pre-amplifier but also a $\Delta \Sigma$ type analog-to-digital converter ( $\Delta \Sigma$ modulator), which has noise shaping capability 9]. The spectroscopic performance has been verified [10], which approved the ASIC as the front-end electronics of the X-ray CCD camera [11] onboard the ASTRO-H mission [12].

The radiation damages to space-use ICs are classified into two types: a total ionization dose (TID) effect and a single event effect (SEE). The former is the accumulating damage mainly due to the geomagnetically trapped protons and electrons. The latter is the stochastic damage that occurs when the energy deposited by a single heavy ion in the Galactic cosmic-rays exceed a threshold defined by the linear energy transfer (LET).

SEE can further be categorized into a single event latchup (SEL) and a single event upset (SEU). SEL is a peculiar issue in CMOS Large Scale Integration (LSI). Since the NMOS and PMOS transistors are formed on a single silicon wafer, there are PNPN junctions forming par- 
asitic thyristors 13]. If the input voltage exceeds that of the power supply momentarily due to the impingement of high energy particle, the thyristor is switched on and the excessive current continues to flow unless the power is shutdown. Finally, the LSI may be broken due to the overheat. On the other hand, SEU is generally represented by the bit inversion in the circuits such as memories and flipflops. Despite the fact that SEU events are not critically harmful to the LSI, it can fudges up false signals.

Nakajima et al. 14] performed the TID test by irradiating our ASICs with a proton beam and verified that the gain and the input equivalent noise were not influenced up to $140 \mathrm{krad}$. The simulated dose rate in the low earth orbit (LEO) of ASTRO-H is $1 \mathrm{krad} / \mathrm{yr}$, which promises no significant accumulating damage during the required mission lifetime of three years. This paper reports the SEE tolerance of the ASIC measured with protons and heavy ions.

After the description of our ASIC and the specification of the SEE test in Section 2, we report the results (Section 3) and the expected SEE rate in the LEO (Section 4), followed by summary (Section 5). Indicated errors below mean $90 \%$ confidence level, unless otherwise mentioned.

\section{Specification of the SEE Test}

\subsection{Specification of the ASIC}

The detailed specification of our ASIC (hereafter we call it MND02) is described in Nakajima et al. [14] and references therein. Here we summarize its characteristics ${ }^{96}$ especially regarding the radiation hardness.

MND02 is equipped with four identical chains that pro- ${ }^{98}$ cess the signals from CCDs simultaneously. Each chain ${ }^{99}$ consists of a preamplifier, a 5-bit digital-to-analog converter (DAC), and two $\Delta \Sigma$ modulators (see Fig. 1 in [14]). ${ }^{100}$ Analog signals from CCD are fed into MND02 through101 AC coupling capacitor. Preamplifier differentially 10 times ${ }^{102}$ amplifies the signals and the 5-bit DAC gives offset to the103 signal level. Two $\Delta \Sigma$ modulators work by turns convertingio4 the amplified analog signals into two 155-bit serial streams 105 15]. Then the bit stream is decimation-filtered in subse-106 quent circuits implemented in another ICs such as field-107 programmable gate arrays (FPGAs) and finally we obtain 108 the 12-bit decimal value. The weighting coefficients for ${ }^{109}$ each bit in the decimation filter has been determined by ${ }^{110}$ simulations (Fi.g 6 in [7]) to enhance the frequency re-111 sponse as a low-pass filter and improve signal-to-noise ra-112 tio.

It was fabricated by Taiwan Semiconductor Manufac-114 turing Company (TSMC) $0.35 \mu \mathrm{m}$ CMOS process through115 MOSIS service. The chip wafer is made from a p-type epi-116 taxial wafer to improve the SEL tolerance. Although we117 do not employ the enclosed layout transistors, all the MOS118 toransistors are surrounded by guard rings to strengthen119 the chip against TID effect. $3 \mathrm{~mm}$ square bare chip is ${ }^{120}$ packed into $15 \mathrm{~mm}$ square ceramic quad flat package (CQFP2).

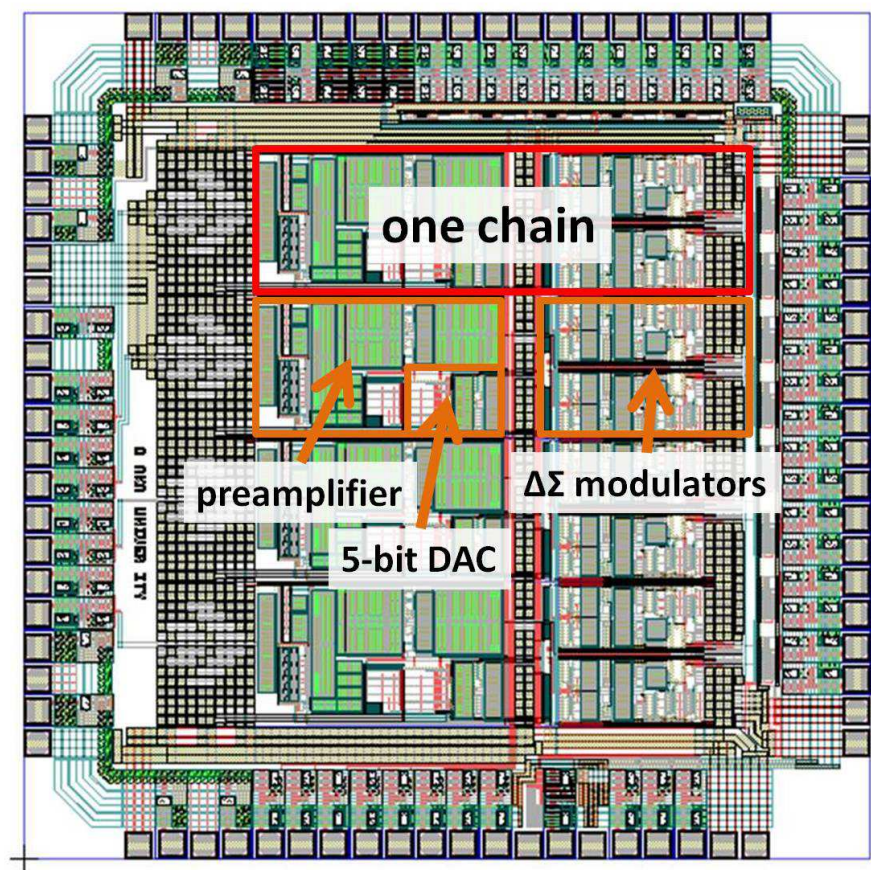

Figure 1: Mask layout of MND02 illustrating the location of the circuit components. The red box surrounds the region of single chain. Four identical chains are located lengthwise. The orange boxes indicate the location of the circuit components in a chain. The size of the chip is $3 \mathrm{~mm}$ square.

It functions with $3.3 \mathrm{~V}$ single power supply for analog and digital circuits. Fig. 1 shows the mask layout of MND02 with the labels of the circuit components. Most of the wafer area is devoted to capacitors.

\subsection{Specification of the heavy ion beam}

We employed the charged-particle beams at a physicsgeneral experiment line 1 (PH1) and the medium energy beam (MEXP) course at Heavy Ion Medical Accelerator at Chiba (HIMAC) in National Institute for Radiological Sciences. Particles are extracted into the MEXP course immediately after the linear accelerator. The particle kinetic energy per atomic mass unit is $6 \mathrm{MeV} / \mathrm{u}$. The beam duration is $350 \mu \mathrm{sec}$ and can be shortened by a chopper down to $5 \mu \mathrm{sec}$. The pulse interval is $1.65 \mathrm{sec}$ and hence the time profile of the beam is quite intermittent. The beam intensity is measured by the Faraday cup (FC) located at the upstream of our chamber. The device under test (DUT) is put in a vacuum state when we use MEXP course to avoid the energy loss and the scattering of the incident particles.

In the case we use PH1 course, the particles are accelerated in the synchrotron ring up to $400 \mathrm{MeV} / \mathrm{u}$ before they are extracted. Since the particles are fed to two rings by turns, the beam interval is $3.3 \mathrm{sec}$, while the duration of the beam on phase is $1 \mathrm{sec}$ at the beam exit. DUTs are in an atmospheric pressure in $\mathrm{PH} 1$ course and are put 
$30 \mathrm{~cm}$ downstream from the beam exit that consists of Aluminum film with the thickness of $100 \mu \mathrm{m}$. The energy loss in the flim and air is well below $0.1 \%$ of the incident energy. Beam fluxes were measured using a scintillation counter prior to each test.

Table 1 lists the specification of the beams adopted in the HIMAC. LET ranges from $5.89 \times 10^{-3}$ to $57.9 \mathrm{MeV} \cdot \mathrm{cm}^{2} / \mathrm{m}$ The beam size was measured by FC located near beam exit and the incident fluxes was derived by fitting the beam profile with a two-dimensional Gaussian model. There is no resin cover above the bare chip and the lid of the CQFP was removed throughout the test to ensure the incident energy. Since the size of the socket on which DUTs are mounted is $50 \mathrm{~mm}$ by $60 \mathrm{~mm}$, the other ICs on the printed circuit board (PCB) were not exposed to the radiation.

We supply $3.3 \mathrm{~V}$ directly from transistor DC power supply unit (PSU) through no serial resistors. Hence the SEL causes immediate current increase up to the limit value. The current in the PCB during test and that of the limit are 0.13 and $0.2 \mathrm{~A}$, respectively. The PSU was programed to shut down immediately when it noticed the current limit and supply voltage again after 5 seconds. We provided pseudo CCD signals into the DUT with a pixel rate of $78 \mathrm{kHz}$ during the test.

\section{Results of SEE Tests}

\subsection{Results of SEL Tests}

Fig.2] shows the time profile of the current in the $\mathrm{PCB}^{180}$ for analog and digital circuit in the case of Xenon beam. ${ }_{182}^{181}$ The signal processing was performed throughout the dura- ${ }_{18}$ tion shown in the figure. The current level during the beam on does not increase compared with that during beam off, which means there is no SEL during the irradiation. Total ${ }_{185}$ fluence of the Xenon ions was $7.2 \times 10^{10} / \mathrm{cm}^{2}$.

The cross-section of the SEL $\left(\sigma_{\mathrm{SEL}}\right)$ is calculated di- ${ }^{186}$ viding the number of the SEL events by the fluence of the ${ }^{187}$ particles. Since there was no SEL event in this experiment, ${ }^{188}$ we estimate the upper limit of $\sigma_{\mathrm{SEL}}$ using Poisson statis- ${ }^{189}$ tics. Assuming three SEL events for the above fluence, the ${ }^{190}$ derived $\sigma_{\text {SEL }}$ is below $4.2 \times 10^{-11} \mathrm{~cm}^{2} /\left(\right.$ Ion $\times$ ASIC) at $95 \%^{191}$ confidence level. Nakajima et al. [14] also performed the ${ }^{192}$ SEL test for MND02 but only for Fe ions with the LET of ${ }^{193}$ $1.68 \mathrm{MeV} \cdot \mathrm{cm}^{2} / \mathrm{mg}$. In this report we put the upper limit of ${ }^{194}$ $\sigma_{\text {SEL }}$ at an LET by 34 times higher than that of the previ- ${ }^{195}$ ous experiment. This LET is sufficiently high enough that ${ }^{196}$ SEL event should not be a cause of instrument downtime. ${ }^{197}$

Although there is no significant increase of the current ${ }^{198}$ due to the beam, some features are seen in Fig. 2. The ${ }^{199}$ fluctuations of the current seen only when the beam was ${ }^{200}$ on can be understood that it is due to the ion-shunt $16,{ }^{201}$ 17, 18]. It often follows from funneling [19, 20] in the ${ }^{202}$ drain depletion layer. When the carriers created by the ${ }^{203}$ charged particle concentrate throughout a volume linking ${ }^{204}$ two junctions, the coupling of the junctions occurs and ${ }^{205}$ then the carriers redistribute. This results in the temporal ${ }^{206}$

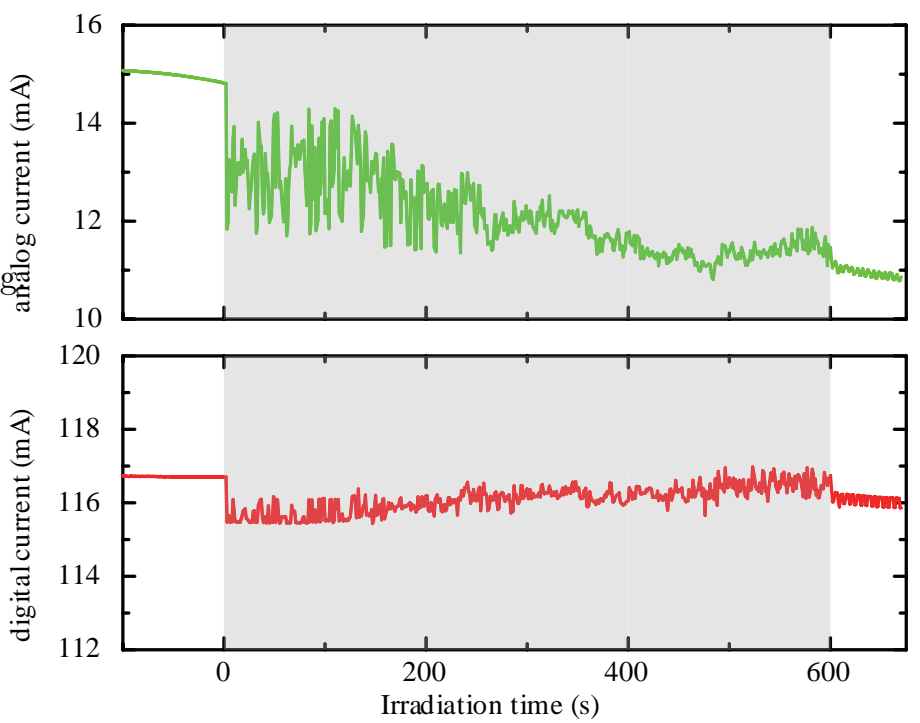

Figure 2: Time profile of the current in the PCB for analog circuit (top panel) and digital circuit (bottom panel) during the beam irradiation of Xenon $6 \mathrm{MeV} / \mathrm{u}$ (hatched duration). The current limit of the PSU is set to be $0.2 \mathrm{~A}$ for the total of analog and digital circuits.

increase of the current in the chip. On the other hand, the current jump down when the beam sets in and the small fluctuations after the beam off cannot be explained by the ion shunt. Some charge up in the chip may explain the former phenomenon. Aside from the fluctuations, there is a slope in the current versus time. This comes from the other ICs than MND02 on the PCB since we see this slope not only in the radiation experiments but also in other experiments.

\subsection{Results of $S E U$ Tests}

Fig. 3 shows the pulse height amplitude (PHA) distributions obtained when we process the pseudo CCD signals of 820 pixels with constant input voltage. While the distribution with beam off can be reproduced with Gaussian, there are some anomalous pixels whose PHA show significantly deviated values from the Gaussian when the Xenon $200 \mathrm{MeV} / \mathrm{u}$ beam is on. We regarded the pixels that deviates more than $4 \sigma$ from the distribution center as SEU events.

The influence of the SEU events on the PHA distribution varies considerably among the circuit components in MND02. If the bit inversion occurs at the resistor in the 5-bit DAC, all the pixels will be affected after the impingement until we set the DAC value again. Assuming that the digital circuit in the final stage of $\Delta \Sigma$ modulator is hit, one of the 155 output bit stream is inverted. Then the expected deviation from the normal PHA depends on the weighting coefficient of the inverted bit and it is no more than $8 \%$ of the normal value [7], which corresponds to 50 units in the case of Fig. 3. In cases where big amounts of electron-hole pairs are created in the capacitors of the preamplifier or the integrator in the $\Delta \Sigma$ modulators, the 
Table 1: Specification of the beams at HIMAC.

\begin{tabular}{lccccc}
\hline Species & $\begin{array}{c}\text { Beam } \\
\text { course }\end{array}$ & $\begin{array}{c}\text { Energy } \\
(\mathrm{MeV} / \mathrm{u})\end{array}$ & $\begin{array}{c}\text { Linear energy transfer } \\
\left(\mathrm{MeV} \cdot \mathrm{cm}^{2} / \mathrm{mg}\right)\end{array}$ & $\begin{array}{c}\text { Beam Width } \\
(\mathrm{mm} \text { in FWHM })\end{array}$ & $\begin{array}{c}\text { Maximum intensity } \\
\left(\mathrm{Ion} / \mathrm{sec} / \mathrm{cm}^{2}\right)\end{array}$ \\
\hline Proton & PH1 & 100 & $5.89 \times 10^{-3}$ & $3.8 \times 1.1$ & $1.7 \times 10^{9}$ \\
Silicon & PH1 & 400 & 0.49 & $2.9 \times 2.6$ & $3.3 \times 10^{4}$ \\
Krypton & PH1 & 200 & 4.72 & $4.4 \times 3.8$ & $3.3 \times 10^{5}$ \\
Iron & PH1 & 400 & 1.68 & $7.1 \times 5.7$ & $3.3 \times 10^{4}$ \\
Xenon & MEXP & 6 & 57.9 & $2.4 \times 1.8$ & $1.0 \times 10^{8}$ \\
& PH1 & 200 & 10.6 & diameter of 10 & $3.3 \times 10^{6}$ \\
& PH1 & 400 & 7.2 & diameter of 10 & $3.3 \times 10^{4}$ \\
\hline
\end{tabular}
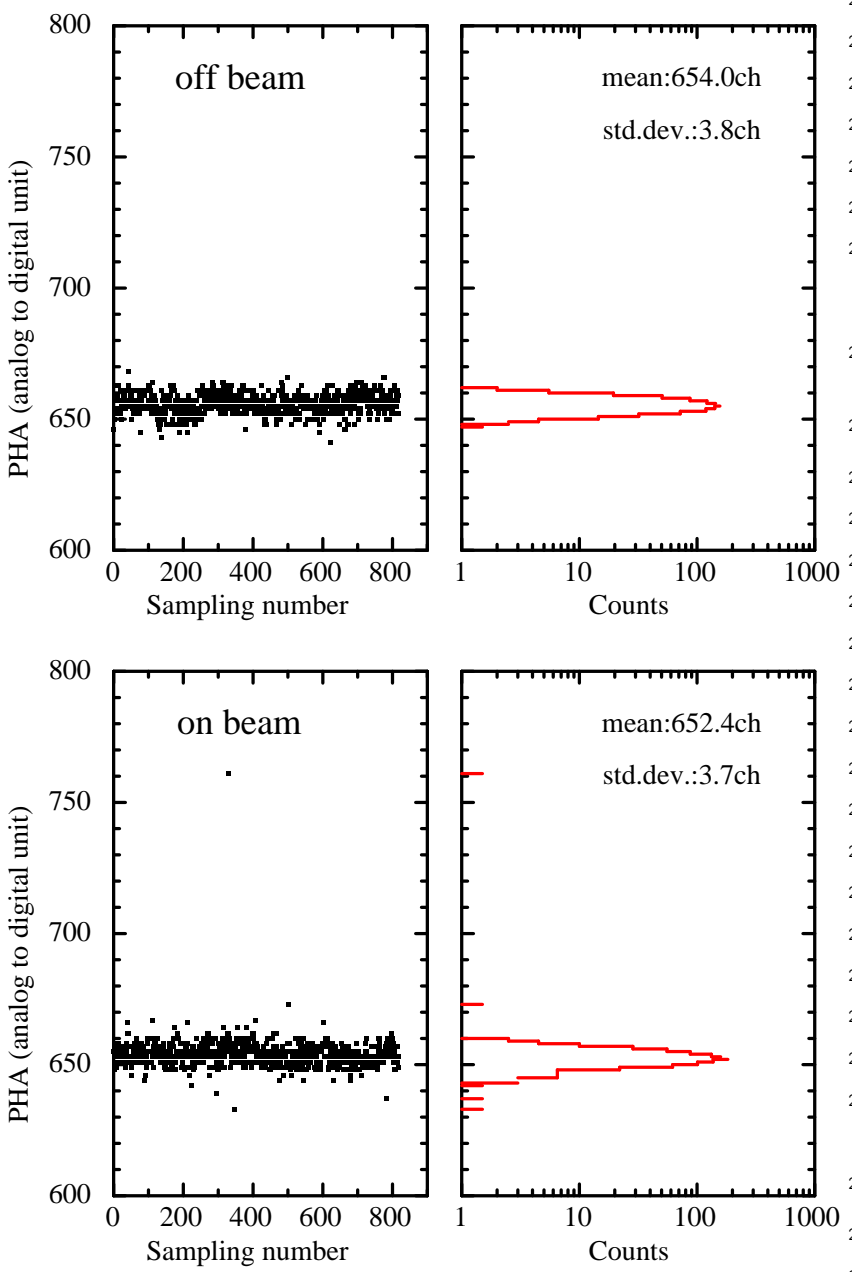

Figure 3: Top panels: PHA distribution of the decimated pseudo 820 signals obtained during beam off and projected histogram of their PHA. Bottom panels: The same as top panels but for the ${ }^{239}$ data obtained during the beam irradiation. Several data points show deviated PHA.

amount of the PHA deviation from the normal value depends on the energy deposited in the chip. Then only the single pixel is affected since we assert a reset signal to both of the preamplifier and the $\Delta \Sigma$ modulator. Considering the fact that the amount of the PHA deviation from the distribution ranges from 20 to 120 units and the fact that the anomalies do not last over pixels, we guess that the probable components affected are the capacitors lying scattered over the chip.

\section{Estimation of the SEE Tolerance}

\subsection{LET spectrum in the LEO}

The SEE event rate of an electronic devices in the specific orbit and duration is estimated by integrating the product of the LET flux and SEE cross-section. The energies, densities and types of particles in the space environment depend significantly on the orbital parameters such as the altitude, the inclination angle, the recent solar activity, and the amount of spacecraft shielding. Then we simulated the LET flux distribution due to the galactic cosmic-rays in the LEO of our primary target mission ASTRO-H using the tools provided by JAXA Space Environment \& Effects System (SEES) group 11, which is shown in Fig.4. In this simulation Cosmic Ray on MicroElectronics (CREME) code 21] is used. In this mission MND02 chips are mounted on a PCB in the camera body that is made of Aluminum with the thickness of about $20 \mathrm{~mm}$.

\subsection{Estimation of SEE rate}

The probability of SEE when a charged particle impact the LSI is expressed as a function of LET $(L)$ as following equation called Weibull curve.

$$
P_{\mathrm{SEE}}(L)=P_{0} \times\left(1-\exp \left(-\frac{L-L_{\mathrm{th}}}{W}\right)\right)
$$

\footnotetext{
${ }^{1}$ http://seesproxy.tksc.jaxa.jp
} 


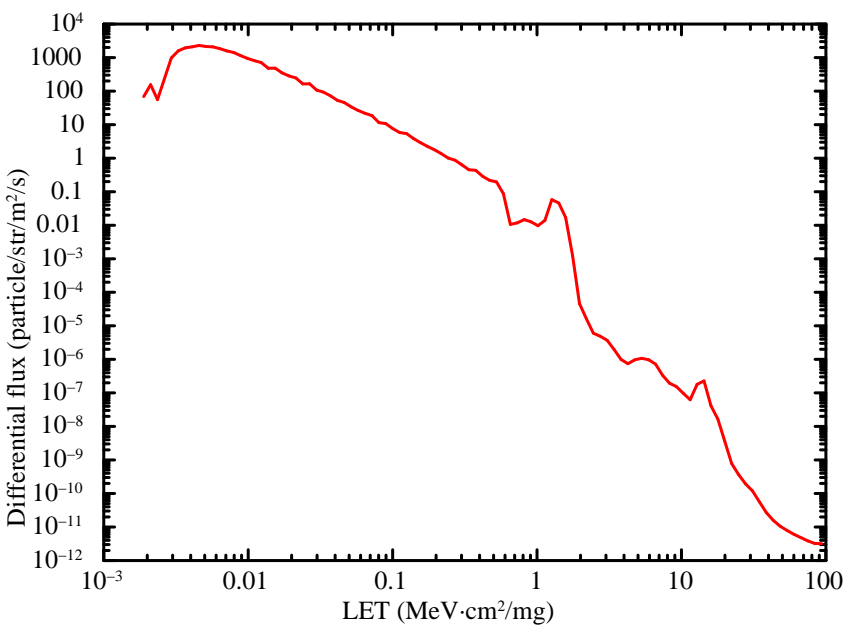

Figure 4: LET spectrum expected in the environment MND02 is located. Assumed orbital parameters are the height of $550 \mathrm{~km}$, the inclination angle of $30^{\circ}$. Aluminum cover with the sickness of $20 \mathrm{~mm}$ surrounds MND02 for all direction. The calculated duration is one year from January 2014 and an influence of magnatic storm is taking into account. where $P_{0}$ is the saturated probability, $L_{\mathrm{th}}$ is the thresh-274 old LET, and $W$ is a curve width. Then we calculate the ${ }_{275}$ SEE rate integrating the product of $P_{\mathrm{SEE}}(L)$ and parti-276 cle flux throughout the available LET range in Fig. $40 \mathrm{of}_{277}$ $2.0 \times 10^{-3} \leq L \leq 100 \mathrm{MeV} \cdot \mathrm{cm}^{2} / \mathrm{mg}$.

Although series resistors are implemented in power lines279 for the flight model electronics to avoid the thermal de-280 struction in the case of the SEL, we require the probabil- ${ }_{281}$ ity for the latch-up to be below once per $30 \mathrm{yrs}$, which ${ }_{282}$ is 10 times the required mission lifetime of ASTRO-H.283 Since we put the upper limit of the SEL probability us- ${ }_{284}$ ing Xenon beam of $6 \mathrm{MeV} / \mathrm{u}$, the most pessimistic estima- ${ }_{285}$ tion of SEL rate is $P_{\mathrm{SEL}}=4.2 \times 10^{-11} \mathrm{~cm}^{2} /(\text { Ion } \times \mathrm{ASIC})_{286}$ for $2.0 \times 10^{-3} \leq L \leq 57.9 \mathrm{MeV} \cdot \mathrm{cm}^{2} / \mathrm{mg}$ and $P_{\mathrm{SEL}}=1$ for $_{287}$ $57.9 \leq L \leq 100 \mathrm{MeV} \cdot \mathrm{cm}^{2} / \mathrm{mg}$. Then the SEL rate is calcu- ${ }_{288}$ lated to be once per $1.5 \times 10^{9} \mathrm{sec}$ or $49 \mathrm{yrs}$, which satisfies ${ }_{289}$ the requirement.

The SEU rate can be estimated in the same manner $\mathrm{as}_{291}$ that for SEL. However, we need an additional care about $_{292}$ the proton data because of the nuclear reactions between ${ }_{293}$ high-energy protons and silicon nuclei in the device. Pro- ${ }_{294}$ tons indirectly induce SEUs since their elastic and inelas- ${ }_{295}$ tic interactions create secondary projectiles. They consist ${ }_{296}$ of neutrons, alpha particles and heavy recoiling ions [22].297 The resultant LET distribution in the silicon wafer is not $_{298}$ a $\delta$-function but wide-spread function.

Hence we desire to calculate the number of SEU events ${ }_{300}$ due to the secondary heavy ions and subtract it from the $e_{301}$ total number of SEUs in the proton test. Since we mea- ${ }_{302}$ sured the SEU probability at the LET of Si $400 \mathrm{MeV} / \mathrm{u}_{303}$ $\left(L_{\mathrm{Si} 400}\right)$, it is assumed that the secondary particles with the LET larger than $L_{\mathrm{Si} 400}$ induce SEUs with the same probability as $P_{\mathrm{SEU}}\left(L_{\mathrm{Si} 400}\right)$. On the other hand, when the

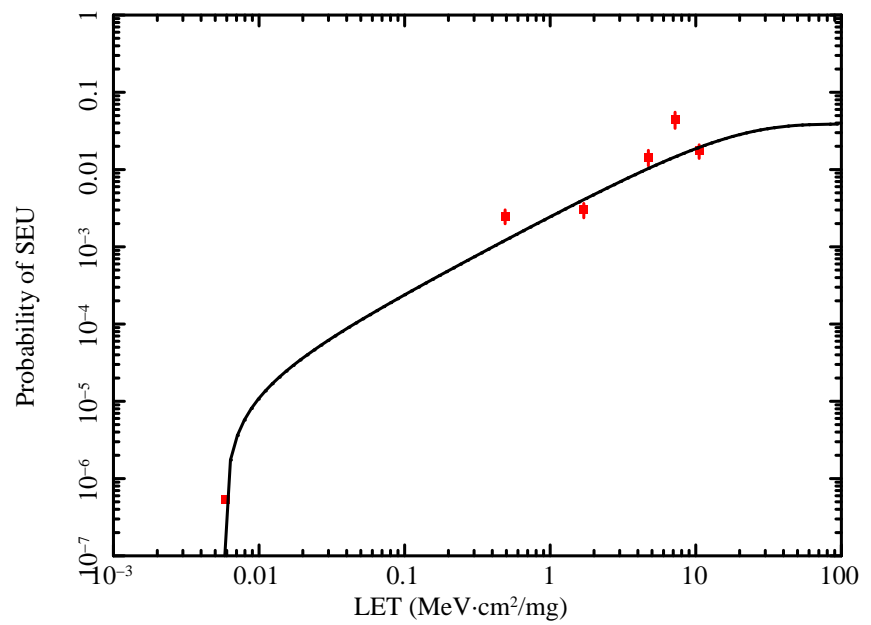

Figure 5: Probability for SEU to occur as a function of LET. The results of 3 DUTs are averaged for each LET, except that we tested 4 chips for proton beam. The recoil component is considered for protons $\left(5.89 \times 10^{-3} \mathrm{MeV} \cdot \mathrm{cm}^{2} / \mathrm{mg}\right)$. Solid line is the Weibull curve fitted with the data.

LET of the secondary particle is smaller than $L_{\mathrm{Si} 400}$, we regard the SEU as that due to the proton. We adopted the LET distribution calculated by Barak 23 in which $4 \times 10^{-5}$ of the total number of protons create the secondary heavy ions whose LETs are larger than $L_{\mathrm{Si} 400}$.

Taking into account the above consideration, we fitted the SEU probability plot with the Weibull function (Fig. (5) . The best fit values are $5.68_{-0.02}^{+0.05} \times 10^{-3} \mathrm{MeV} \cdot \mathrm{cm}^{2} / \mathrm{mg}$ for $L_{\mathrm{th}}, 3.886 \pm 0.001 \times 10^{-2}$ for $P_{0}$, and $15.3_{-9.7}^{+166.6} \mathrm{MeV} \cdot \mathrm{cm}^{2} / \mathrm{mg}$ for $W$. We then estimated the SEU event rate using fitted parameter to be $8.0_{-7.3}^{+13.9} \times 10^{-6} \mathrm{SEU} / \mathrm{sec}$.

Nevertheless the sharpness of the rising edge in Weibull function strongly depends on this estimation, the number of the data below $1 \mathrm{MeV} \cdot \mathrm{cm}^{2} / \mathrm{mg}$ is limited in our experiment. Therefore we estimate the systematic error as follows considering that the Weibull function is monotonically increasing. In the worst case estimation, the probability of SEU in an LET range higher than a data point and lower than the next one is assumed to be that of the latter data point. In this way we estimated most pessimistic rate to be $1.3 \times 10^{-3} \mathrm{SEU} / \mathrm{sec}$.

Most of the SEU events such as that in Fig. 3 cannot be distinguished from the signals due to X-ray photons from astronomical objects. This means we need to consider the SEU event as a part of the non X-ray background (NXB). The NXB intensity of the X-ray CCD Camera onboard ASTRO-H is expected to be similar to that of the X-ray Imaging Spectrometer onboard Suzaku satellite, which is 0.1 counts/sec [24]. Since the upper limit of the SEU rate is below $1.3 \%$ of known NXB rate, it should not be a major component of the instrumental background. 


\section{Conclusion}

We have developed an mixed-signal ASIC for the read-362 out circuit of the onboard CCD camera. The results of the ${ }^{363}$ radiation tolerance test for SEE using protons and heavy ${ }_{365}^{364}$ ion beams can be summarized as follows

1. We detected no SEL events even with a high $\mathrm{LET}_{368}^{367}$ beam of Xenon $6 \mathrm{MeV} / \mathrm{u}$. The upper limit of the ${ }^{369}$ cross-section is $\sigma_{\mathrm{SEL}}<4.2 \times 10^{-11} \mathrm{~cm}^{2} /($ Ion $\times \mathrm{ASIC}){ }_{371}^{370}$ The flapping of the current seen only when the beam ${ }_{372}^{371}$ was on can be understood such that it is due to the $\mathbf{s}_{373}$ ion-shunt.

2. Some anomalous pixels that have peculiar PHA are arf $_{376}^{375}$ seen only when the beam is on, which we regard ${ }_{377}$ as SEU events. The most probable location of the ${ }^{378}$ impingement is capacitors in the preamplifier $\operatorname{and}^{379}$ the integrator of the $\Delta \Sigma$ modulators.

3. The SEE event rate in an LEO is estimated by inte- 382 grating the product of the simulated LET flux spec- ${ }^{383}$ trum and SEE probability. The upper limit of the ${ }_{385}^{384}$ SEL rate is once per 49 years, which satisfies our $_{386}$ requirement. The upper limit of the SEU rate is de-387 rived to be $1.3 \times 10^{-3}$ events/sec, which is $1.3 \%$ of $^{388}$ known NXB rate.

\section{Acknowledgment}

We acknowledge people of Accelerator Engineering Corporation, especially Satoshi Kai who offered us much supports in the radiation damage experiment. We used the simulation tools provided by SEES group in JAXA. This work is supported by the Nano-Satellite Research and Development Project in Japan, the Research Project with Heavy Ions at NIRS-HIMAC, and the JSPS KAKENHI Grant Number 22740122, 23000004, and 24684010.

\section{References}

[1] M. C. Weisskopf, B. Brinkman, C. Canizares, G. Garmire, S. Murray, L. P. Van Speybroeck, Publ. Astron. Soc. Pacific, $114(2002) 1$

[2] F. A. Jansen and XMM Science Operations Team, American Astronomical Society 32 (2000) 724

[3] K. Mitsuda, et al., Publ. Astron. Soc. Japan 59 (2007) 1

[4] R. Rando, A. Bangert, D. Bisello, A. Candelori, P. Giubilato, M. Hirayama, R. Johnson, H. F. -W. Sadrozinski, M. Sugizaki, J. Wyss, M. Ziegler, IEEE Trans. Nucl. Sci., 51 (2004) 1067

[5] H. Tajima, T. Nakamoto, T. Tanaka, S. Uno, T. Mitani, Ed. Ce. Silva, Y. Fukazawa, T. Kamae, G. Madejski, D. Marlow, K. Nakazawa, M. Nomachi, Y. Okada, T. Takahashi, IEEE Trans. Nucl. Sci., 51 (2004) 842

[6] S. Herrmann, W. Buttler, R. Hartmann, P. Holl, N. Meidinger, L. Strueder, Nucl. Sci. Symposium Conference Record IEEE vol.3 (2007) 2398

[7] D. Matsuura, H. Nakajima, E. Miyata, H. Tsunemi, J. P. Doty, H. Ikeda, Proc. SPIE 6686 (2007) 66860L

[8] H. Nakajima, D. Matsuura, N. Anabuki, E. Miyata, H. Tsunemi, J. P. Doty, H. Ikeda, T. Takashima, H. Katayama, IEEE Trans. Nucl. Sci. 56 (2009) 747

[9] H. Inose, Y. Yasuda, J. Murakami, IRE Transactions on Space Electronics Telemetry SET-8 (1962) 204
[10] H. Nakajima, D. Matsuura, N. Anabuki, E. Miyata, H. Tsunemi, J. P. Doty, H. keda, H. Katayama, Nucl. Instr. and Meth. A 610 (2009) 78

[11] K. Hayashida et al., Proc. SPIE 8145 (2011) 814505

[12] T. Takahashi et al., Proc. SPIE 7732 (2010) $77320 \mathrm{Z}$

[13] B. L. Gregory, B. D. Shafer, IEEE Trans. Nucl. Sci. 20 (1973) 293

[14] H. Nakajima, D. Matsuura, T. Idehara, N. Anabuki, H. Tsunemi, J. P. Doty, H. Ikeda, H. Katayama, H. Kitamura, Y. Uchihori, Nucl. Instr. and Meth. A 632 (2011) 128

[15] J. P. Doty, D. Matsuura, H. Ozawa, E. Miyata, H. Tsunemi, H. Ikeda, Proc. SPIE 6276 (2007) 62761P

[16] J. R. Hauser, S. E. Diegl-Nagle, A. R. Knudson, A. B. Campbell, W. J. Stapor, P. Shapiro, IEEE Trans. Nucl. Sci. 32 (1985) 4114

[17] A. R. Knudson, A. B. Campbell, J. R. Hauser, M. Jessee, W. J. Stapor, P. Shapiro, IEEE Trans. Nucl. Sci. 33 (1986) 1560

[18] A. O. Brown, B. Bhuva, S. E. Kerns, W. J. Stapor, IEEE Trans. Nucl. Sci. 40 (1993) 1918

[19] C. M. Hsieh, P. C. Murley, R. R. O'Brien, IEEE Electron Device Lett. 2 (1981) 103

[20] A. B. Campbell, A. R. Knudson, W. J. Stapor, P. Shapiro, S. E. Diehl, J. Hauser, IEEE Trans. Nucl. Sci. 32 (1985) 4128

[21] J. H. Adams, Jr., R. Silberberg, C. H. Tsao, Navy Research Laboratory Memorandum Report 4506 (1981)

[22] A. Akkerman, J. Barak, J. Levison, Y. Lifshitz, Radiation Physics and Chemistry 48 (1996) 11

[23] J. Barak., IEEE Trans. Nucl. Sci. 48 (2001) 1937

[24] K. Koyama et al., Publ. Astron. Soc. Japan 59 (2007) 23 